\title{
Performance of Electrolyte Composed of Spiro-type Quaternary Ammonium Salt and Electric Double-layer Capacitor Using It
}

\author{
Kazumi ChiBa, * Tsukasa Ueda, and Hideo Yamamoto
}

Japan Carlit Corporation, R\&D center (Shibukawa, Gunma 377-0004, Japan)

Received January 30, 2007 ; Accepted May 5, 2006

\begin{abstract}
An electrolyte composed of a tetra-alkyl ammonium cation having a spiro structure (spiro-(1,1')-bipyrrolidinium: SBP) showed excellent performance in terms of electric conductivity, viscosity, and solubility in various solvents, especially propylenecarbonate (PC). The electric double-layer capacitor (EDLC) using spiro-(1,1')-bipyrrolidinium tetrafluoroborate in $\mathrm{PC}\left(\mathrm{SBP}_{-} \mathrm{BF}_{4} / \mathrm{PC}\right)$ has a performance better than that using tetraethylammonium tetrafluoroborate in $\mathrm{PC}\left(\mathrm{TEA}-\mathrm{BF}_{4} / \mathrm{PC}\right)$ and triethylmetylammonium tetrafluoroborate in PC (TEMA-BF $\left.4 / \mathrm{PC}\right)$, especially at low temperatures and high discharging rates.
\end{abstract}

Key Words : Electric Double-Layer Capacitor, Electrolyte, Spiro-type Quaternary Ammonium Salt

\section{Introduction}

Recently, large electric double-layer capacitors (EDLCs) have been developed for hybrid cars, ${ }^{1)}$ uninterruptible power sources (UPSs) ${ }^{2}$ and other systems. Even though the performance of an electrolyte strongly affects the performance of a capacitor, no new organic liquid electrolyte has been developed that is better than the propylenecarbonate (PC) solution of asymmetrical-structure quaternary tetra-alkyl ammonium tetrafluoroborate, for example, triethylmethylammonium tetrafluoroborate in $\mathrm{PC}$ (TEMA-BF $\mathrm{T}_{4} / \mathrm{PC}$ ), and a traditional symmetricalstructure analogue namely, tetraethylammonium tetrafluoroborate in $\mathrm{PC}\left(\mathrm{TEA}-\mathrm{BF}_{4} / \mathrm{PC}\right)$.

In an earlier study, Morimoto et al. (1987) showed that an asymmetrical-structure quaternary ammonium salt exhibits a better performance as an electrolyte of EDLC ${ }^{3)}$ than a symmetrical-structure analogue. They reported that the asymmetrical-structure salt dissolved well in PC; this characteristic gave a low internal resistance to the EDLC.

Ammonium salts with various structures were examined by Ue et al. (1994), including spiro-structure salts.) In their paper, the performances of electrolytes, for

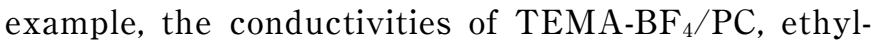
methylpyrrolidinium tetrafluoroborate in PC (EMP$\mathrm{BF}_{4} / \mathrm{PC}$ ) and spiro-(1,1')-bipyrrolidinium tetrafluoroborate in $\mathrm{PC}\left(\mathrm{SBP}-\mathrm{BF}_{4} / \mathrm{PC}\right)$, were reported to be good, because of their smaller ion sizes. However, they did not show in detail the performances of EDLCs that use these electrolytes.

Among the three analogues, TEMA-BF $4 / \mathrm{PC}$ was only commercialized later, because it seemed to have the highest potential of being synthesized at a low cost. On the other hand, $\mathrm{SBP}_{-} \mathrm{BF}_{4}$ has not been of interest until now. We report the characteristics of $\mathrm{SBP}_{-} \mathrm{BF}_{4} / \mathrm{PC}$, which according to $\mathrm{Ue}$ et al. has the best conductivity because the ion dissociation level is higher than that of TEMA-BF ${ }_{4} / \mathrm{PC}$.

\section{Experimental}

Although $\mathrm{SBP}_{-\mathrm{BF}_{4}}$ is expected to have excellent characteristics, the method of synthesizing SBP-BF ${ }_{4}$ has not been reported. The procedure is shown below. Pyrrolidine $(329.3 \mathrm{~g}: 5.00 \mathrm{~mol})$ was added dropwise to the isopropanol $(2,500 \mathrm{~g})$ solution of 1,4-dibromobutane $(1,079.6 \mathrm{~g}: 5.00 \mathrm{~mol})$ and potassium carbonate $(829.3 \mathrm{~g}$ : $6.00 \mathrm{~mol}$ ) with stirring in an ice bath. After dropwise addition, reflux was conducted for $5 \mathrm{~h}$ at $356 \mathrm{~K}$. Then the solution was filtered (ADVANTEC 5C) for the removal of potassium bromide; isopropanol was removed by evaporation at $343 \mathrm{~K}$ under reduced pressure. The salt was washed twice with $1,000 \mathrm{ml}$ of acetone to remove byproducts. The prepared spiro-(1,1')-bipyrrolidinium bromide (SBP-Br) was dried under vacuum for $12 \mathrm{~h}$ at 353 K (yield: $89 \%$ ).

The SBP-Br was dissolved in water (1,000 g) and made to react with silver oxide $(579.4 \mathrm{~g}: 2.5 \mathrm{~mol})$ to obtain spiro-(1,1')-bipyrrolidinium hydroxide (SBP-OH) (yield: $99 \%)$.

The obtained SBP-OH was made to react with 42 wt.\% $\mathrm{HBF}_{4} \mathrm{aq}$, and water was removed by evaporation at $343 \mathrm{~K}$ under reduced pressure to obtain $\mathrm{SBP}_{-} \mathrm{BF}_{4}$ salt (yield: $96 \%$ ). $\mathrm{SBP}^{-\mathrm{BF}_{4}}$ was assigned by ${ }^{1} \mathrm{H}$ NMR $(300$ $\left.\mathrm{MHz}, \mathrm{CD}_{3} \mathrm{OD}, 298 \mathrm{~K}\right): \delta=2.18-2.24(\mathrm{~m}, 8 \mathrm{H}), 3.50-3.57(\mathrm{~m}$, $8 \mathrm{H})$.

The obtained SBP-BF 4 was then dissolved in PC; moleculer sieves (TOHSO Co., 8-10mesh, type: 3A) were added to dry the electrolytic solution for 2 weeks at 253 K. After dehydration (residual water: $<50 \mathrm{ppm}$ ), the electrolytic solution was filtered using a disposable filter unit (ADVANTEC Co., pore size: $0.50 \mu \mathrm{m}$ ) in dry Ar atmosphere.

Coconut shell charcoal (surface area: $1,500 \mathrm{~m}^{2} / \mathrm{g}$ ) $85 \%$, Ketjen black (Lion Co., ECP-600JD) 5\%, and polytetrafluoroethylene $45 \%$ ethanol solution (Mitsui-Dupont florochemical Co., 30J) $20 \%$ were mixed, ground, and pressed to form a disk composite $15 \mathrm{~mm}$ in diameter and $0.8 \mathrm{~mm}$ 
thick. A pair of these disk composite electrodes was dried at $473 \mathrm{~K}$ below $2,500 \mathrm{~Pa}$ for $12 \mathrm{~h}$, and then immersed in electrolytes under reduced pressure. Using these electrodes and a polypropylene separator and gasket unit, a 2032 coin cell was assembled.

The electric conductivity of the electrolyte was measured using a conductivity meter equipped with a standard conductivity cell (Toa Electronics, CM-20J/C50101B) and refrigerated/heating circulators (Julabo Co., FP50). Its viscosity was measured using a viscosity meter (SUN Science Co., Rheo Meter CR-500DX) and FP50. The potential window was measured by cyclic voltammetry with an automatic polarization system (Hokuto Denko Co., HZ-3000). The counterelectrode was Pt wire, the working electrode consisted of glassy carbon rods, and the reference electrode was $\mathrm{Ag} / \mathrm{Ag}^{+}$. The scan speed was $20 \mathrm{mV} / \mathrm{s}$, and the temperature of dry Ar atmosphere was $298 \mathrm{~K}$.

The performance of EDLC was measured using a charge/discharge unit with a high speed logger (Hokuto Denko Co., HJ1001SM8B) and a thermal shock chamber (ESPEC Co., TSA-40C). The capacitance $C$ of the assembled cell was determined by charge-discharge cycling between 0 and $2.7 \mathrm{~V}$ at a constant current $I$ of $5 \mathrm{~mA}$ at a given temperature, where it was calculated from discharging time $t$ using $C=I t / V$. The specific capacitance was obtained by dividing the above capacitance by the total weight of a pair of the disk electrodes. The internal resistance was calculated from the IR drop when the discharge current was $50 \mathrm{~mA}$ at a given temperature. The specific internal resistance was obtained by dividing the above internal resistance by the total weight of a pair of the disk electrodes. The capacitances $C$ at various discharging rates were calculated from discharging $(2.7 \mathrm{~V}$ to $0 \mathrm{~V})$ at various currents $I(2,5,10,2050,100,200,500$, $900 \mathrm{~mA})$. The specific capacitance at various discharging rates was obtained by dividing the above capacitance by the area of the disk electrode. All data are the average values of three equivalent cells.

\section{Results and Discussion}

The most important characteristic of an electrolyte is the solubility to the solvent, because the maximum conductivity of the electrolytic solution is affected by the solubility of the electrolyte in the solvent. A high conductivity gives EDLCs a low internal resistance. Table 1 shows the solubility of TEA-BF 4 , TEMA-BF 4 and SBP$\mathrm{BF}_{4}$ in $\mathrm{PC}$ at $303 \mathrm{~K}$. It shows that $\mathrm{SBP}_{-} \mathrm{BF}_{4}$ has the highest solubility. $\mathrm{SBP}_{-\mathrm{BF}_{4}}$ as a spiro-type electrolyte, whose molecular structure has a characteristic ' 8 ' shape, having a little symmetry, has a higher solubility in PC than asymmetrical-structure TEMA-BF 4 and symmetricalstructure TEA-BF 4 . In particular, the solubility of SBP$\mathrm{BF}_{4}$ in $\mathrm{PC}$ is three times as high as that of TEA-BF 4 . This shows that the spiro-structure differs markedly from 'true' symmetrical-structure (TEA-BF ${ }_{4}$ ).

Figure 1 shows the electric conductivity of three types of electrolytic solution at $303 \mathrm{~K}$. The maximum conductivity of SBP is higher than those of TEA and TEMA, because of the good solubility of SBP-BF 4 in PC. At the

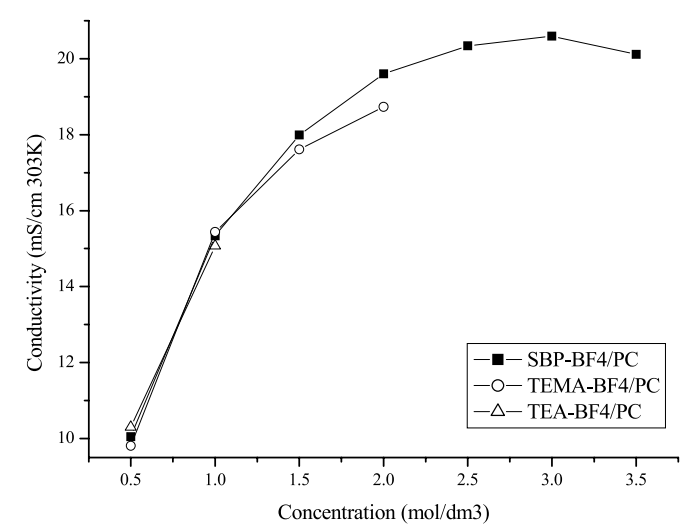

Fig. 1 Relationship between conductivity and concentration at $303 \mathrm{~K}$.

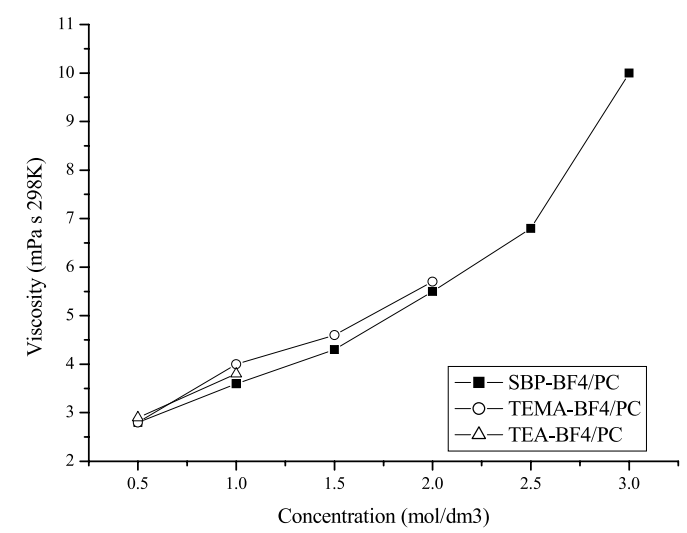

Fig. 2 Relationship between viscosity and concentration at $298 \mathrm{~K}$.

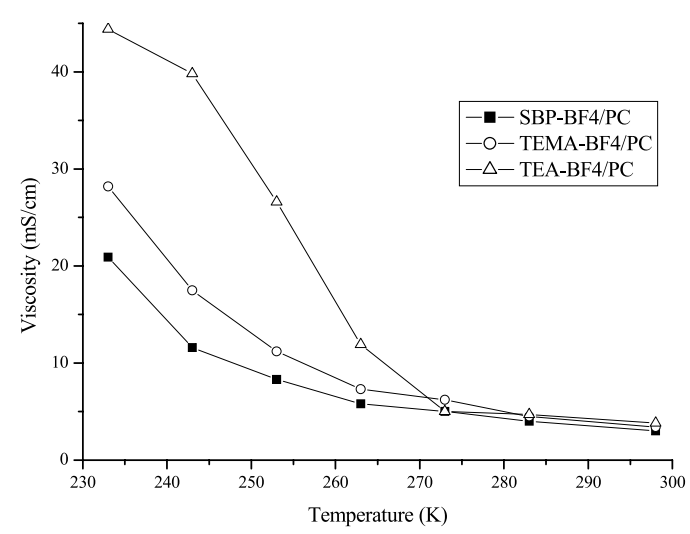

Fig. 3 Relationship between viscosity and temperature for $1.0 \mathrm{~mol} / \mathrm{dm}^{3}$ electrolytes.

same salt concentration, the electric conductivity of SBP$\mathrm{BF}_{4} / \mathrm{PC}$ is better than those of TEA-BF $/ \mathrm{PC}$ and TEMA$\mathrm{BF}_{4} / \mathrm{PC}$, because SBP cations may have a smaller volume than TEA and TEMA cation structures and have a high mobility in the electrolyte, which were not examined.

In addition, the electric conductivity is affected by the viscosity of the electrolytic solution. Figure 2 shows that the relationships between the viscosity and the salt con- 

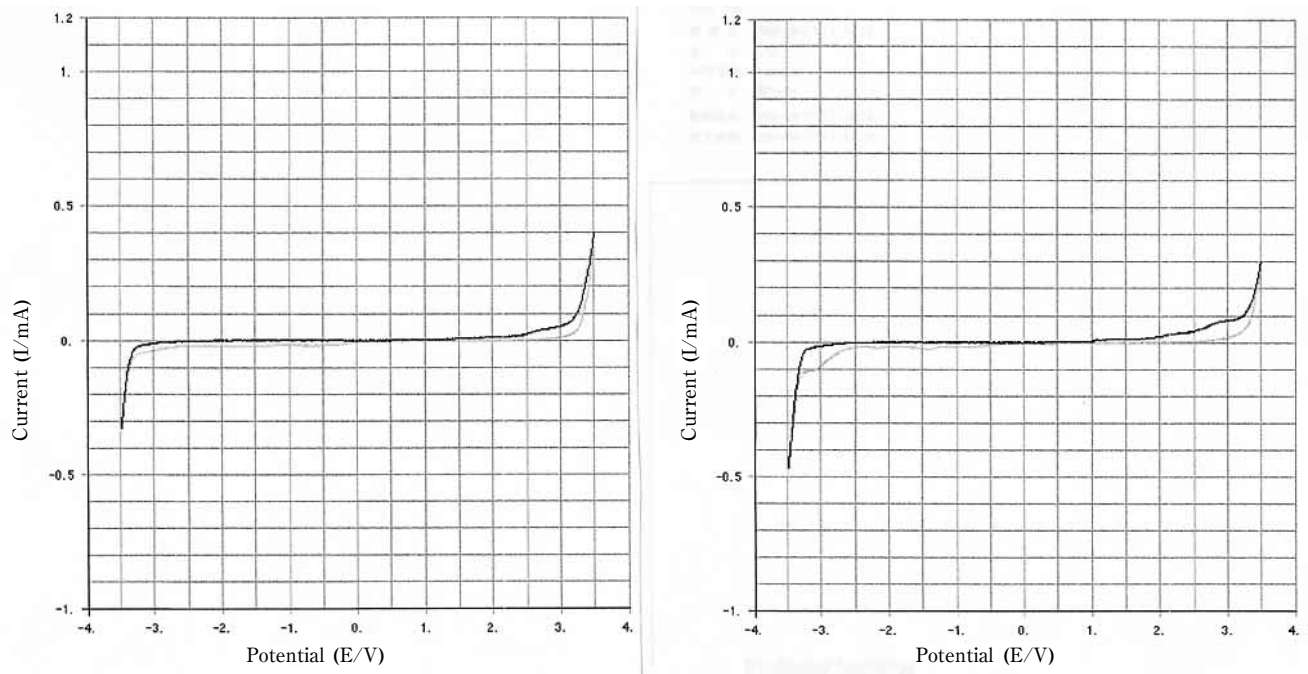

Fig. $4 \mathrm{CV}$ curves of $1.0 \mathrm{~mol} / \mathrm{dm}^{3} \mathrm{SBP}_{-} \mathrm{BF}_{4} / \mathrm{PC}$ and TEA-BF $4 / \mathrm{PC}$ on a glassy carbon electrode at $298 \mathrm{~K}$.

Table 1 Solubility of each salt in PC at $303 \mathrm{~K}$.

\begin{tabular}{cccc}
\hline & SBP-BF $_{4}$ & TEMA-BF $_{4}$ & TEA-BF $_{4}$ \\
\hline $\begin{array}{c}\text { Solubility } \\
\left(\mathrm{mol} / \mathrm{dm}^{3}\right)\end{array}$ & 3.55 & 2.21 & 1.10 \\
\hline
\end{tabular}

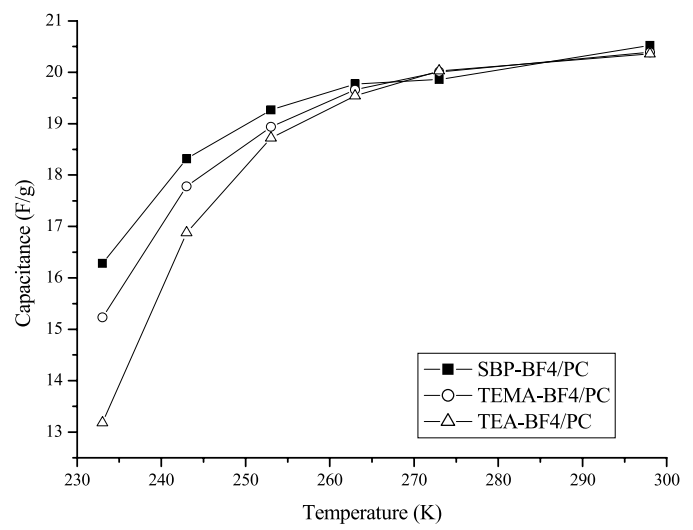

Fig. 5 Relationship between capacitance and temperature for $1.0 \mathrm{~mol} / \mathrm{dm}^{3}$ electrolytes.

centration for TEA, TEMA and SBP electrolytes; they are similar to each other at the practical level (0.5-2.0 $\mathrm{mol} / \mathrm{dm}^{3}$ ). However, because this measurement had carried out at $298 \mathrm{~K}$, the measurement temperature was changed. The viscosity of TEA-BF $4 / \mathrm{PC}$ rapidly increased at low temperatures below about $253 \mathrm{~K}$, because TEA$\mathrm{BF}_{4} / \mathrm{PC}$ began to freeze. Furthermore, the viscosity of SBP was lower than that of TEMA in a wide temperature range (Fig. 3); the difference in salt solubility in PC, shown in Table 1, may be exhibited. As regards electrochemical stability, the potential window of $1.0 \mathrm{~mol} / \mathrm{dm}^{3}$ $\mathrm{SBP}-\mathrm{BF}_{4} / \mathrm{PC}$ electrolyte was the same as that of 1.0 $\mathrm{mol} / \mathrm{dm}^{3}$ TEA-BF $4 / \mathrm{PC}$ as shown in Fig. $4 . \mathrm{SBP}^{-B_{4}}$ did not have a problem regarding electrochemical stability, in contrast to TEA-BF . $_{4}$

Figure 5 shows the relationship between the capaci-

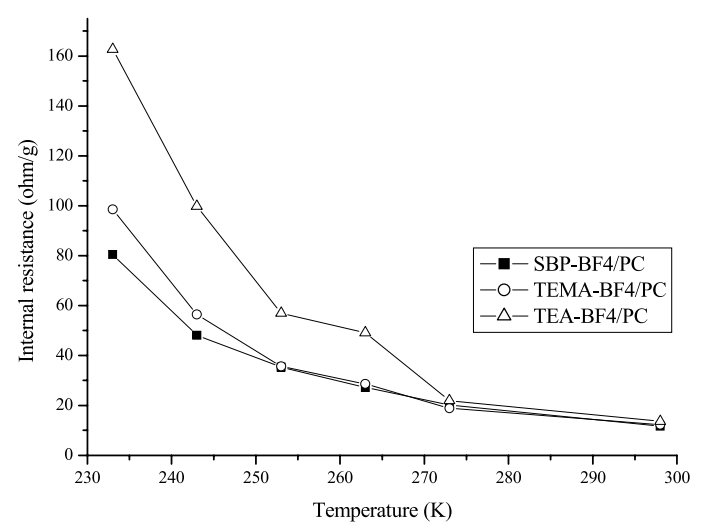

Fig. 6 Relationship between internal resistance and temperature for $1.0 \mathrm{~mol} / \mathrm{dm}^{3}$ electrolytes.

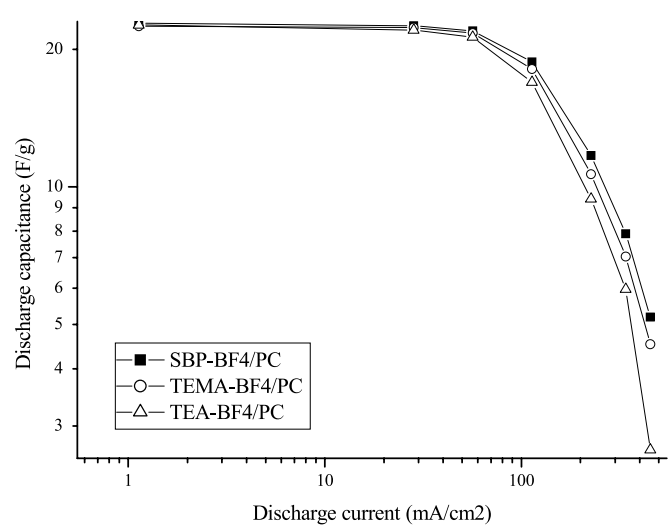

Fig. 7 Relationship between discharge capacitance and discharge current at $298 \mathrm{~K}$.

tance of EDLC and the type of electrolyte. At room temperature, the capacitance of EDLC using SBP electrolyte was larger than those of EDLCs using TEA and TEMA. At low temperatures, the difference increased.

In the same manner, Fig. 6 shows that the internal resistance of EDLC using SBP was smallest for all tem- 
peratures. The cause of the difference may be the volume of the cations and the viscosity of the electrolyte (Fig. 3). The shape of the curves in Fig. 6 is similar to that in Fig. 3. Therefore, it shows that the viscosity of electrolytic solution has a very large effect on the characteristics of EDLCs especially at low temperatures.

The capacitance of EDLC using SBP electrolytes did not decrease at high discharging rates compared with those of TEA and TEMA (Fig. 7). This result shows that the mobility of SBP ions in the electrolyte is high compared with those of TEA and TEMA ions in PC. The measurement was carried out at $298 \mathrm{~K}$. At the temperature, there was no difference in viscosity for these types of electrolytic solutions (Fig. 3); thus, the reason for the difference may be the small size and rigidity of the structure of SBP ions compared with those of TEA and TEMA. However, we do not understand this in detail.

\section{Conclusion}

1. An electrolyte composed of SBP-BF 4 and PC showed excellent performance features of high conductivity, and low viscosity over a wide temperature range.

2. $\mathrm{SBP}_{-} \mathrm{BF}_{4}$ did not have a problem regarding electro- chemical stability compared with TEA-BF 4 .

3. An EDLC using SBP-BF $F_{4} / \mathrm{PC}$ had a large capacitance and a low internal resistance over a wide temperature range. In particular, at low temperatures, the EDLC showed excellent performance.

4. The viscosity of electrolytic solution had a very large effect on the characteristics of EDLCs especially at low temperatures.

5. The capacitance of EDLC consisting of SBP electrolyte did not decrease as much as those of EDLCs consisting of TEA and TEMA at high discharging rates.

\section{Acknowledgement}

We are grateful to Professor Yuichi Sato of Kanagawa University for his generous support.

\section{References}

1) M. Sasaki, Electrochemistry, 72, 772 (2004).

2) T. Muto, Electrochemistry, 72, 775 (2004).

3) T. Morimoto, K. Hiratsuka, M. Sanada, and H. Ariga, Japanese, 358526 (1987).

4) M. Ue, K. Ida, and S. Mori, J. Electrochem. Soc., 141, 2989 (1994). 